\title{
Latent inhibition of the conditioning context: Further evidence of contextual potentiation of retrieval in the absence of appreciable context-US associations
}

\author{
MARY ANN BALAZ, SHARON CAPRA, WESLEY J. KASPROW, \\ and RALPH R. MILLER \\ State University of New York, Binghamton, New York
}

\begin{abstract}
Previous research in our laboratory has found superior performance when classically conditioned responses are observed in the training context as opposed to outside it, even when direct context-US associations have been minimized by either the choice of conditioning parameters or extinction to the context. The present experiment used latent inhibition of the conditioning context as an alternative method of examining contextual cue effects in the absence of appreciable direct context-US associations. Water-deprived rats received tone-footshock pairings in one of two distinctly different apparatuses, but all were tested in a common apparatus. Animals conditioned in the test enclosure displayed more lick suppression than those conditioned outside the test enclosure. Other animals tested without the tone present also exhibited more suppression if conditioning had occurred in the test context rather than outside it, indicating that direct associations between the conditioning context and shock had been formed. However, when formation of direct associations to the conditioning context was attenuated in additional animals through extensive preexposure to the context prior to conditioning, more suppression to the tone was still seen when conditioning had occurred in the test context rather than outside it. These results add support to the position that the training context augments recall even when direct associations between the context and the US are attenuated. The phenomenon is discussed in terms of facilitated retrieval of nominal CS-US associations, configural retrieval cues, and conditional discriminations.
\end{abstract}

Recall of a specific memory ordinarily is greater when testing occurs in a surrounding similar to that of original learning. This enhanced performance in the presence of the training context exemplifies the "contextual cue effect" which has been repeatedly observed, using both human and animal subjects (e.g., Smith, 1979; Zentall, 1970). Although the benefit to performance of testing in the training context has been known for quite some time (e.g., Pan, 1926), the mechanisms underlying the effect of context on the manifest retention of specific target events have been examined only recently (e.g., Bouton \& Bolles, 1979a, 1979b; Spear, Smith, Bryan, Gordon, Timmons, \& Chiszar, 1980).

The explanation most commonly put forth for the enhanced performance observed when testing occurs at the site of original learning is that the training context acts as a conditioned or discriminative stimulus (CS or SD) in its own right, and its associative strength

This research was supported by NIMH Grant 33881 and NIH BRSG Grant S07RR07149-06. Thanks are due Todd Schachtman for critically commenting on an early draft of the manuscript. Reprint requests should be addressed to Ralph R. Miller, Department of Psychology, SUNY, Binghamton, New York 13901. summates with that of the nominal CS or SD (Rescorla \& Wagner, 1972; Wagner, 1978). In the case of classical conditioning, it is generally believed that associations are formed not only between the nominal CS and the unconditioned stimulus (US), but also between other cues in the environment, that is, the context, and the US. Thus, the enhanced performance seen when testing occurs in the conditioning context is the result of a larger number of direct associations to the US contributing to the test performance. Put in other terms, performance outside the training environment is assumed to be attenuated because context-US associations are not activated. Considerable data give credence to this view, particularly the occurrence of conditioned responses (CRs) in the training context prior to presentation of the nominal CS.

On the other hand, Konorski (1967), Medin (1975), and Spear (1973) have suggested that the training context "potentiates" retrieval of associations between the nominal CS and US, that is, the presence of the conditioning context during testing enhances the effectiveness of the nominal CS in eliciting conditioned responding. (This use of the term "potentiation" here is similar to, but should not be con- 
fused with, potentiation of acquisition which ordinarily is the converse of overshadowing.) More precisely, Medin (1975) and Spear (1973) hypothesize that the context serves as a cue for the retrieval of associations to the nominal $\mathrm{CS}$ as the result of its being an attribute of the target memory system. Nadel and Willner (1980) advocate a similar view, specifically that a hierarchical relationship of cues exists in which contexts both contain and predict CSs. These potentiation-of-retrieval interpretations of the contextual cue effect do not exclude the summation view; rather, both factors in principle could contribute to the observed phenomenon.

Balaz, Capra, Hartl, and Miller (1981) recently presented data illustrating the contribution of contextual potentiation of retrieval to the superiority of conditioned responding in the presence of the training context. They gave water-deprived rats toneshock pairings in one of two distinct enclosures and then extinguished the context-US associations in some of the animals by giving them extended exposure to the apparatus cues prior to testing. Nonextinguished animals tested in the absence of the tone displayed more suppression of baseline licking in the conditioning context than outside it, attesting to the presence of direct context-US associations. However, even in the absence of appreciable associations to the conditioning context following extinction to the context, animals tested in the conditioning context showed better retention of the tone-shock association than animals tested in a different context. This finding suggests that although direct associations to contextual cues when present may contribute to the contextual cue effect, there is a further factor contributing to the effect, for example, potentiation of retrieval. In another study, Balaz et al. (1981) employed conditioning parameters that prevented the formation of appreciable context-US associations. This was accomplished by using a large number of CS-US pairings (and correspondingly decreasing US intensity) relative to experiments in which formation of context-US associations was evident. That such manipulations minimize direct context-US associations has been demonstrated by Odling-Smee (1978) and is consistent with the Rescorla-Wagner (1972) model, which predicts that contextual cues should first gain and then, due to intertrial extinction, lose associative strength as the number of conditioning trials increases. A contextual cue effect for the target CS was still observed by Balaz et al., even though the formation of appreciable context-US associations was prevented. Although these results support the notion of potentiated retrieval of conditioned associations to the US when testing occurs in the conditioning context, further demonstrations of potentiation of retrieval using other means of eliminating direct associations to the context are necessary to establish the generality of the phenomenon.
The present experiment was designed to prevent the formation of context-US associations by exposing animals to the conditioning context prior to CS-US pairings; that is, a latent inhibition (LI) paradigm was employed. Latent inhibition, first reported by Lubow and Moore (1959), refers to the retarded development of a $C R$ that results from repeated exposure to a potential CS prior to the CS-US pairings. It has been assumed that this preconditioning exposure to the CS interferes with the formation of associations between the to-be-conditioned stimulus and the US.

Although a variety of explanations have been offered for the latent inhibition phenomenon (for a review, see Lubow, 1973), its basis is not crucial to our present concern with the contextual cue effect. What is of interest is that latent inhibition to the conditioning context potentially can attenuate responding arising from direct context-US associations that would otherwise summate with responding dependent upon associations between the nominal CS and US. Such a reduction in the influence of direct contextUS associations would reduce the summative component of the contextual cue effect, thereby revealing any residual element of the contextual cue effect that might be attributable to potentiation of retrieval by the conditioning context. Notably, we do not yet understand the mechanisms underlying the hypothesized potentiation-of-retrieval effect; conceivably, preconditioning exposure to the context impairs contextual potentiation of retrieval as rapidly as or more rapidly than it retards acquisition of context-US associations. Therefore, any failure to observe contextual potentiation of retrieval following such a procedure would not provide strong evidence against contextual potentiation of retrieval contributing to the contextual cue effect. On the other hand, observation of a contextual cue effect after using latent inhibition of the conditioning context to prevent an appreciable contribution of direct context-US associations would support the existence of contextual potentiation of retrieval as a factor in producing the contextual cue effect.

This possibility provided the impetus for the present experiment, in which water-deprived rats were used as subjects in a Pavlovian lick-suppression paradigm. The animals were first allowed to drink from lick tubes in each of two distinctly different environments ( $Y$ and $Z$ ). Half of the animals received extended exposure to each apparatus ( $\mathrm{LI}$ treatment) prior to receiving tone(T)-footshock pairings in either enclosure $Y$ or enclosure $Z$. The remainder of the animals received a short exposure to each apparatus (SH treatment) prior to conditioning. Pilot studies had indicated that $4 \mathrm{~min}$ of prior exposure to the test enclosure was sufficient for an animal to produce short latencies to begin licking on a test trial, and that less than $6 \mathrm{~h}$ of preconditioning exposure was not 
effective in attenuating the acquisition of context-US associations.

In addition to testing for the amount of suppression to the nominal CS, as assessment of associations to background cues was also necessary to determine the effectiveness of our latent inhibition manipulation. However, the anticipated relatively low levels of fear evoked by these contextual cues might prove difficult to detect through suppression of an operant baseline; that is, following latent inhibition to the context, a floor effect might obscure residual conditioning of context-US associations that could, however, become manifest when superimposed on associations between the nominal CS and US (Reberg, 1972). Consequently, we sought a means of elevating baseline suppression during tests for direct associations to the context. This was accomplished by giving all animals additional conditioning trials consisting of distinctive clicks paired with footshock in a third apparatus (X), prior to tone-footshock pairings. Later, when testing animals for direct associations to the conditioning context in the absence of the tone, the clicks were presented, thereby producing an elevated suppression baseline around which small amounts of fear to the test apparatus could summate. This procedure increased the sensitivity to detect any existing low levels of fear to contextual cues.

\section{METHOD}

\section{Subjects}

Fifty-three experimentally naive male Sprague-Dawley-derived rats from the SUNY-Binghamton breeding colony, approximately 70 days of age at the beginning of the study, were used as subjects. The rats were housed in individual wire-mesh cages with free access to food and, starting about 2 weeks prior to the beginning of the experiment, were placed on a water-deprivation schedule (10 min per day) and handled daily. The vivarium was maintained on a 16-h/8-h light/dark cycle. The animals were assigned randomly to one of eight groups, with somewhat more animals being placed in those groups that yielded relatively high within-group variance in the first two of five replications; however, all groups were represented in each replication.

\section{Apparatus}

The conditioning apparatus consisted of two types of enclosures ( $Y$ and $Z$ ) which differed radically from each other. In all, six identical $Y$ enclosures and six identical $Z$ enclosures were used. Groups were as closely balanced with respect to specific enclosures as possible in light of the slightly unequal $\mathbf{n}$ between groups. Enclosure $\mathrm{Y}$ was a clear Plexiglas box measuring $22.75 \times 8.25 \times$ $13.00 \mathrm{~cm}(1 \times \mathrm{w} \times \mathrm{h})$ with a narrow-diameter, stainless steel grid floor $(.48-\mathrm{cm}$-diam rods spaced $1.91 \mathrm{~cm}$ apart center-to-center) joined by NE- 2 neon bulbs through which a $1.0-\mathrm{mA}$ constant current footshock could be administered. Each $Y$ enclosure was housed in an environmental chamber illuminated by a $7.5-\mathrm{W}$, 120-V incandescent bulb, with one speaker (above the box) providing a high-frequency buzzing background noise $(60 \mathrm{dBC}$, re $20 \mu \mathrm{N} / \mathrm{m}^{2}$ ) and another speaker (below the box) for delivery of the CS, a $725-\mathrm{Hz}, 70-\mathrm{dBC}$ tone. A block of wood, treated daily with six drops of methyl salicylate, provided a distinctive odor in enclosure $Y$. A lick tube was located at one end of the apparatus $3.8 \mathrm{~cm}$ above the grid floor.

Enclosure $Z$ was housed in a different type of environmental chamber and consisted of an unlighted 30-cm-long box made of stainless steel and black Plexiglas in a truncated $\mathrm{V}$ shape. The metal interior was $28 \mathrm{~cm}$ high, $5.25 \mathrm{~cm}$ wide at the bottom, and $21.50 \mathrm{~cm}$ wide at the top. The floor consisted of two parallel metal plates separated by a $1.25-\mathrm{cm}$ gap. Both the sloping walls and the floor were capable of being electrified to deliver 1.0-mA constantcurrent shock. A lick tube was located at the end of the enclosure $1.50 \mathrm{~cm}$ above the floor. A background white noise $(60 \mathrm{dBC})$ was provided through an overhead speaker, and a second speaker below floor level was available to deliver the same tonal CS as in enclosure Y.

A third apparatus (enclosure $X$ ), distinctly different from both enclosure $Y$ and enclosure $Z$, was used for pairing clicks and footshock. Enclosure X was a $50 \times 25 \times 59 \mathrm{~cm}(1 \times \mathrm{w} \times \mathrm{h})$ box with a large-diameter $(.64 \mathrm{~cm})$ stainless steel grid floor $(1.59 \mathrm{~cm}$ center-to-center). One long wall was made of aluminum and the remaining three were opaque Plexiglas. The grid floor was connected through NE-2 neon bulbs to a shock generator that could administer a 1.0-mA constant current footshock. A speaker located centrally below the box provided $3 / \mathrm{sec}$ clicks at $5 \mathrm{dBC}$ above a 62 -dBC hissing background noise supplied via a speaker located on the ceiling of the room. The duration of orienting behavior of pilot animals to the $\triangle 5-\mathrm{dBC}$ clicks on initial presentation was highly similar to that to the $\triangle 10-\mathrm{dBC}$ tone, suggesting that the two stimuli were of approximately equal saliency. In addition, human observers found the clicks as well as the tone to sound highly similar across enclosures $X, Y$, and $Z$. Apparatus $X$ was located in a separate, brightly illuminated room that was quite distinct from the room housing enclosures $\mathrm{Y}$ and $\mathrm{Z}$.

\section{Procedure}

For half the animals, lick tube shaping (Days 1-4) consisted of a short (SH) daily exposure, of 1-min duration, to enclosures $Y$ and $Z$, each with its lick tube present. For the other half of the animals, latent inhibition (LI) to enclosures $Y$ and $Z$ was incorporated into lick tube shaping on Days $1-4$ by extending daily exposure to enclosures $\mathrm{Y}$ and $\mathrm{Z}$ to $1 \mathrm{~h}$ each with the lick tubes present. Lick-shaping sessions consisted of placing each animal in an enclosure facing away from the lick tube and recording its latency to complete 50 licks via a lickometer circuit. Lick-tube training was terminated after $1 \mathrm{~min}$ or after $1 \mathrm{~h}$ regardless of the number of licks emitted. The two exposures (one each to enclosures $\mathrm{Y}$ and $Z$ ) were separated by a 2 -h interval, with the order of exposures being alternated over days and counterbalanced within groups. To approximately equate the level of deprivation between the two groups of animals, those animals which received only a 1 -min exposure to the two different enclosures received $1 \mathrm{~h} /$ day access to water in the home cages, whereas LI animals, which were given $1 \mathrm{~h} /$ day of exposure to the two enclosures with water available, received no water in the home cages on days during which they experienced these prolonged preconditioning exposures to enclosures $\mathrm{Y}$ and $\mathrm{Z}$.

On Day 5, all animals were exposed to clicks paired with footshock in enclosure X. During the 12.7-min conditioning session, each animal received 12 pairings of an $18-\mathrm{sec}$ train of clicks and a 2-sec footshock, with an intertrial interval of $40 \mathrm{sec}$. Onset of the US was concurrent with the offset of the clicks. Two hours following completion of conditioning to the clicks, the LI groups received an additional $1-h$ exposure to both enclosure $Y$ and enclosure $Z$. The two exposures were again separated by $2 \mathrm{~h}$ spent in the home cage, and lick tubes were present during these exposures. The SH groups remained in their home cages during this period and received $1 \mathrm{~h}$ of access to water.

On Day 6, LI animals received their final preconditioning exposure to enclosures $\mathrm{Y}$ and $\mathrm{Z}$, conducted in the same manner as on Day 5 , bringing the total amount of time spent by each LI ani$\mathrm{mal}$ in each apparatus (Y and $\mathrm{Z}$ ) to $6 \mathrm{~h}$. Two hours after the conclusion of this last latent inhibition treatment, all subjects received conditioning to the tone $(T)$, the target $C S$. Each rat was placed in its designated $\mathrm{Y}$ or $\mathrm{Z}$ enclosure for $12.7 \mathrm{~min}$, with the lick tube removed. All animals received 12 tone-footshock pairings, with the tone having an 18-sec duration, the footshocks having a 2-sec 
duration, and tone offset being concurrent with shock onset. An interval of $\mathbf{4 0} \mathrm{sec}$ separated the trials. Following the conditioning trials, each animal was returned to its home cage.

On Day 7, all subjects were tested in enclosure $Y$. Thus, half of the animals were tested in the same context in which conditioning had occurred, whereas the remaining animals were tested outside their conditioning context. It should be noted that, by manipulating conditioning enclosure rather than test enclosure, we avoided the possibility of differential generalization from enclosure X's influencing performance on the test trials. Any generalization from enclosure $X$ to enclosure $Y$ should have been equivalent for all groups. In addition, half of the animals were tested with the target CS (tone) present, whereas the other animals were tested in the presence of the clicks that had been paired with shock on Day 5. This design yielded a total of eight groups. A summary of the treatment given each group is presented in Table 1. Group names summarize the treatments animals received. The first two letters represent the duration of preconditioning exposure to enclosures $\mathrm{Y}$ and $\mathrm{Z}$, with $\mathrm{SH}$ indicating short exposure and LI indicating long exposure, that is, latent inhibition. The third letter signifies the apparatus in which conditioning took place, enclosure $Y$ or enclosure $Z$, and the fourth letter indicates the stimulus presented during testing, either clicks $(C)$ or tone $(T)$. Recall that the clicks were intended to elevate baseline suppression in order to ensure that any low-level fear in response to the apparatus cues could be detected by virtue of summation with fear evoked by the clicks. Testing consisted of placing an animal in the appropriate enclosure facing away from the lick tube with either the target CS or clicks present. Latency to complete 50 licks was recorded. A ceiling latency of 3,600 sec was imposed, at which time testing was terminated and a latency of $3,600 \mathrm{sec}$ was recorded for any subject who met this criterion. In practice, only one animal suppressed for the entire duration of the test.

Based on these manipulations, lick suppression was anticipated as a result of (1) direct tone-shock or click-shock associations in all animals, (2) direct enclosure Y-shock associations in those animals that were conditioned in enclosure $\mathrm{Y}$ without extensive prior exposure to enclosure Y (Groups SHYC and SHYT), (3) residual nonassociative neophobia to enclosure $\mathrm{Y}$ in all animals that had not had extensive prior exposure to enclosure Y (all SH groups), and (4) potentiated retrieval of shock associations by the conditioning context in animals tested with the tone, provided conditioning had occurred in the test context (Groups SHYT and LIYT) and provided extensive pretraining exposure to the conditioning apparatus does not interfere with this presumed potentiation.

\section{RESULTS}

Tested with the clicks present, LI animals displayed equal suppression regardless of where they re- ceived tone-shock pairings, whereas $\mathrm{SH}$ animals displayed greater suppression if they received toneshock pairings in enclosure $Y$ than if they received tone-shock pairings in enclosure $\mathrm{Z}$. With the tone present during testing, both $\mathrm{LI}$ and $\mathrm{SH}$ animals showed more suppression as the result of conditioning in enclosure $Y$ than as a result of conditioning in enclosure $Z$.

All latency scores were converted to log (base 10) latencies to allow the use of parametric statistics. By Day 4, all animals were emitting 50 licks in each of the two lick enclosures in substantially less than $1 \mathrm{~min}$. There were no differences between enclosures $\mathrm{Y}$ and $\mathrm{Z}$ in the latency to complete 50 licks (means = 1.10 and $1.15 \log \mathrm{sec}$, respectively, for $\mathrm{SH}$ animals and means $=1.17$ and $1.07 \log \mathrm{sec}$, respectively, for LI animals).

A three-way analysis of variance (ANOVA) for unequal $n$ (Keppel, 1972) was performed on the data from the Day 7 test with treatment (LI vs. SH), context ( $\mathrm{Y}$ vs. $\mathrm{Z}$ ), and test stimulus (tone vs. click) as the between-group factors forming a $2 \times 2 \times 2$ design. The analysis revealed a significant three-way interaction between treatment, context, and test stimulus $[F(1,45)=7.33, p<.01]$. An analysis of the simple interaction effects at different levels of treatment indicated that a significant context $\times$ test stimulus interaction existed for animals that had received the latent inhibition treatment $[F(1,45)=7.43, p<.01]$, but not for animals that did not receive latent inhibition $[F(1,45)=1.27, p>.20]$. Planned comparisons between pairs of means revealed that $\mathrm{SH}$ subjects suppressed reliably more both to the tone and the click when tone conditioning had occurred in the test context (enclosure $Y$ ) than when tone conditioning had occurred outside the test context [enclosure Z; Groups SHYT vs. Group SHZT, $t(10)=3.89$, and Group SHYC vs. Group SHZT, $t(9)=2.76$, both ps < .05]. In contrast, subjects receiving latent inhibition treatment displayed no difference in suppression as a function of conditioning location when tested with the clicks present [Group LIYC vs.

Table 1

\begin{tabular}{|c|c|c|c|c|c|c|c|}
\hline \multirow[b]{2}{*}{ Group } & \multirow[b]{2}{*}{$\mathrm{n}$} & \multirow{2}{*}{$\begin{array}{c}\text { Latent } \\
\text { Inhibition* }\end{array}$} & \multirow{2}{*}{$\begin{array}{c}\text { Click } \\
\text { Conditioning }\end{array}$} & \multirow{2}{*}{$\begin{array}{c}\text { Tone } \\
\text { Conditioning }\end{array}$} & \multirow{2}{*}{$\begin{array}{c}\text { Test } \\
\text { Condition }\end{array}$} & \multicolumn{2}{|c|}{ Test Suppression $†$} \\
\hline & & & & & & Mean & SE \\
\hline SHYC & 5 & & $12 \mathrm{CX}+$ & $12 \mathrm{TY}+$ & $\mathrm{CY}$ & 3.10 & .08 \\
\hline SHZC & 6 & & $12 \mathrm{CX}+$ & $12 \mathrm{TZ}+$ & CY & 2.19 & .29 \\
\hline SHYT & 5 & & $12 \mathrm{CX}+$ & $12 \mathrm{TY}+$ & TY & 3.17 & .10 \\
\hline SHZT & 7 & & $12 \mathrm{CX}+$ & $12 \mathrm{TZ}+$ & TY & 2.75 & .07 \\
\hline LIYC & 7 & $Y, Z$ & $12 \mathrm{CX}+$ & 12 TY+ & $\mathrm{CY}$ & 1.53 & .27 \\
\hline LIZC & 8 & $Y, Z$ & $12 \mathrm{CX}+$ & $12 \mathrm{TZ}+$ & $\mathrm{CY}$ & 1.62 & .24 \\
\hline LIYT & 7 & $\mathrm{Y}, \mathrm{Z}$ & $12 \mathrm{CX}+$ & $12 \mathrm{TY}+$ & TY & 2.77 & .14 \\
\hline LIZT & 8 & $\mathrm{Y}, \mathrm{Z}$ & $12 \mathrm{CX}+$ & $12 \mathrm{TZ}+$ & TY & 1.67 & .25 \\
\hline
\end{tabular}

Note-C = clicks; $T=$ tone; $X, Y, Z=$ three distinctly different contexts; $S H=$ short preconditioning exposure to enclosures $Y$ and $Z ; L I=$ long preconditioning exposure (latent inhibition) to enclosures $Y$ and $Z ;+=1-m A$ 2-sec footshock. *Six 1 h exposures to enclosure $Y$ and enclosure $Z$ (one session after lick shaping on each of Days 1-4, one after click conditioning on Day 5 , and one prior to tone conditioning on Day 6). TMean latency to complete 50 licks, with standard error (log sec). 
Group LICZ, $\mathrm{t}(13)=.25, \mathrm{p}>.50$ ]; however, LI animals tested with the tone present did display more suppression if they had been conditioned in enclosure $Y$ than if conditioning had occurred in enclosure $Z$ [Group LIYT vs. Group LIZT, $t(13)=3.65$, $\mathrm{p}<.01]$. This indicates that, although the extended exposure to the lick contexts prior to conditioning resulted in no appreciable direct associations between the context and shock being formed during Day 6 conditioning, suppression to the tone was still enhanced by the contextual cues that were present during the tone-footshock pairings.

\section{DISCUSSION}

These results demonstrate that, although direct associations between contextual cues and footshock may occur (e.g., Group SHYC vs. Group SHZC), they are not necessary for the augmented CR typically seen when testing occurs in the training context. Even after latent inhibition had minimized the formation of direct associations between the conditioning enclosure and footshock, a significant contextual cue effect was observed with respect to the tone. This finding is consistent with previous studies (Balaz et al., 1981) in which the prevention or elimination of conditioning-context/US associations, by use of suitable parameters or extinction, respectively, also failed to eliminate the enhanced CR seen when testing was conducted in the same place as conditioning had occurred as opposed to elsewhere. Thus, there appears to be a process giving rise to the contextual cue effect over and above the summation of direct context-US associations with direct associations between the nominal CS and the US.

When discussing the possibility of associations between the training context and the US, we have limited the discussion to the formation of first-order associations. However, Nelson and Friedrich (1980) provide evidence that second-order associations, acquired through mediation by the nominal CS, can be established between the context and the US. Although second-order associations to the context cannot be excluded as a contributing component to contextual cue effects in general, it seems unlikely that appreciable second-order associations would have been formed in our LI groups given the extensive preexposure to the conditioning context experienced by these animals. At the empirical level, the lack of any difference in suppression between Group LIYC and Group LIZC stands as evidence that latent inhibition to the context did effectively prevent the formation of second-order as well as first-order associations to the conditioning context; yet, a contextual cue effect was seen in Group LIYT relative to Group LIZT.

One possible view of the observed contextual cue effect in the LI animals is that the observed differ- ences arose from a reduction of suppression in enclosure $\mathrm{Y}$ when it was the nonconditioning apparatus, as opposed to an enhancement of suppression in enclosure $\mathrm{Y}$ when it was the conditioning apparatus. Such a reduction could conceivably arise as a result of drinking-compatible responses activated by contextual cues constituting enclosure $\mathrm{Y}$ in those animals that had never received footshock there, thereby contributing to the lesser suppression seen in Group LIZT than Group LIYT. However, the lack of any differences between Groups LIYC and LIZC argues against enclosure $\mathrm{Y}$ cues having a suppressionattenuating effect on animals not receiving tone-shock pairings there. Similarly, the lack of difference between Groups LIYC and LIZC argues against enclosure Y's having become a conditioned inhibitor for animals receiving footshock in enclosures $X$ and $Z$ but not enclosure $\mathrm{Y}$.

Another approach to the data would be to analyze them in terms of contemporary theories of conditioning. However, the Rescorla and Wagner (1972) model has no mechanism for changing the associability of a stimulus as a result of nonreinforced presentations and consequently does not predict the latent inhibition which clearly occurred in the present study (LIYC vs. SHYC). On the other hand, attentional theories of conditioning (e.g., Mackintosh, 1975; Pearce \& Hall, 1980) predict that preconditioning exposure to the contextual cues would result in a loss of attention to the context and a corresponding increase in attention to the tone. This effect should have allowed the LI animals to form stronger toneshock associations than the SH animals. Because of the possibly attenuating effects of $\mathrm{LI}$ treatment upon nonassociative fear of enclosures $\mathrm{Y}$ and $\mathrm{Z}$ (neophobia), direct comparisons between $\mathrm{SH}$ and LI groups tested outside their conditioning contexts are inappropriate to evaluate this prediction of the attentional hypothesis. However, if LI treatment did shift growth in associative strength from the contextual cues to the tone, this should have reduced the difference between Groups LIYT and LIZT relative to that between Groups SHYT and SHZT. In fact, the former difference is greater than the latter, and the highlatency cutoff imposed during testing argues against this relationship's being due to a ceiling effect that would artificially reduce the difference between Groups SHYT and SHZT. Thus, the data suggest that an additional factor beyond the scope of the attentional model influenced behavior.

Because other explanations of the enhanced suppression exhibited by LI animals in the presence of conditioning context appear to be inadequate, we favor the explanation put forth by Medin (1975) and Spear (1973). They hypothesize that the conditioning context facilitates retrieval of associations to the US as the result of the context's being an attribute of the target memory system. 
Neither Medin (1975) nor Spear (1973) detailed a mechanism for the contextual potentiation effect that they hypothesized, although they did point out correctly that, as the enhanced performance is dependent on a previously learned association with performance variables presumably held constant, the attenuated performance seen when testing occurs outside the conditioning context is a retrieval deficit. The basis for enhanced retrieval of US associations in the training context is not clear, and identifying the contextual potentiation effect as being the result of facilitated retrieval does not illuminate the specific underlying mechanisms, but we feel that it may have heuristic value. There appear to be at least three means by which testing in the conditioning context could facilitate retrieval of associations that would increase suppression. The one favored by Medin and Spear is that, by some unidentified process, the conditioning context potentiates retrieval of the association between the nominal CS and footshock. Alternatively (or in addition to this), contextual potentiation of retrieval could arise from the contextual cues and nominal CS, jointly constituting a configured discriminative stimulus which, in its own right, becomes associated to footshock in addition to any existing context-footshock and tonefootshock associations. Although the present data do not differentiate these two variations of the potentiation position, the configural hypothesis at least depends upon previously observed processes, whereas potentiated retrieval of tone-footshock associations by the conditioning context evokes a seemingly new and undocumented process.

As a third variation on the potentiation-of-retrieval concept, the contextual cue effect observed in our latent inhibition animals could be viewed as an example of a conditional discrimination, with the apparatus cues playing the role of conditioned discriminative stimuli. This seems particularly plausible given the extensive opportunity that latent inhibition animals had to learn that enclosures $Y$ and $Z$ were different. Although a number of hypotheses concerning the nature of conditional discriminations have been proposed (e.g., Asratian, 1972; Carter \& Werner, 1978), their bases are not well understood. Nevertheless, it is possible that the same processes that give rise to conditional discriminations are also responsible for the potentiating effects of the training context. There are at least two apparent differences between conditional discriminations and contextual potentiation that argue against the latter's being a subset of the former. First, discrete $S$ - trials were not required for the contextual potentiation of retrieval of CS-US associations, whereas they are believed to be necessary for the acquisition of conventional conditional discriminations. Second, contextual potentiation-of-retrieval effects appear to be acquired quite rapidly, as is evident from the present and pre- vious data (Balaz et al., 1981), whereas conditional discriminations typically are formed over many trials. If neither discrete $S-$ trials nor large numbers of training trials are actually essential for the formation of conditional discriminations, then an argument could be made that the contextual potentiation effect is a special case of conditional discrimination. But, even if this proves to be true, it is possible that either facilitated retrieval of nominal CS-US associations and/or retrieval of US associations to the configured CS-context might prove in the end to be the most parsimonious explanation of conditional discriminations in general as well as contextual cue effects in particular. Thus, viewing the contextual cue effect as an instance of conditioned discrimination is not necessarily inconsistent with the first two explanations of the contextual potentiation-of-retrieval phenomenon. Further research will be required to differentiate between these mechanisms. However, the present data serve as further evidence that the contextual cue effect is a consequence of some sort of contextual potentiation of retrieval in addition to direct context-US associations.

\section{REFERENCES}

Asratian, E. A. Genesis and localization of conditioned inhibition. In R. A. Boakes \& M. S. Halliday (Eds.), Inhibition and learning. London: Academic Press, 1972.

Balaz, M. A., Capra, S., Hartl, R., \& Miller, R. R. Contextual potentiation of acquired behavior after devaluing direct context-US associations. Learning and Motivation, 1981, 12, 383-397.

Bouton, M. E., \& Bolles, R. C. Contextual control of the extinction of conditioned fear. Learning and Motivation, 1979, $10,445-466$. (8)

Bouton, M. E., \& Botles, R. C. Role of conditioned contextual stimuli in reinstatement of extinguished fear. Journal of Experimental Psychology: Animal Behavior Processes, 1979, 5, 368-378. (b)

Carter, D. E., \& Werner, T. J. Complex learning and information processing by pigeons: A critical analysis. Journal of the Experimental A nalysis of Behavior, 1978, 29, 565-601.

KE PPEL, G. Design and analysis: $A$ researcher's handbook. Englewood Cliffs, N.J: Prentice-Hall, 1973.

KONORSKI, J. Integrative activity of the brain: An interdisciplinary approach. Chicago: University of Chicago Press, 1967.

Lubow, R. E. Latent inhibition. Psychological Bulletin, 1973, $79,398-407$.

Lubow, R. E., \& Moore, A. U. Latent inhibition: The effect of nonreinforced preexposure to the conditioned stimulus. Journal of Comparative and Physiological Psychology, 1959, 52, 415-419.

Mackintosh, N. J. A theory of attention: Variations in the associability of stimuli with reinforcement. Psychological Review, 1975, 82, 276-298.

Medin, D. L. A theory of context in discrimination learning. In G. H. Bower (Ed.), The psychology of learning and motivation (Vol. 9). New York: Academic Press, 1975.

Nadel, L., \& Willner, J. Context and conditioning: A place for space. Physiological Psychology, 1980, 8, 218-228.

Nelson, D. L., \& Friedrich, M. A. Encoding and cuing sounds and senses. Journal of Experimental Psychology: Human Learning and Memory, 1980, 6, 717-731.

Odling-Smee, F. J. The overshadowing of background: Some effects of varying amounts of training and UCS duration. 
Quarterly Journal of Experimental Psychology, 1978, 30, 737. 746.

PAN, S. The influence of context upon learning and recall. Journal of Experimental Psychology, 1926, 9, 468-491.

Pearce, J. M., \& Hall, G. A model for Pavlovian learning: Variations in the effectiveness of conditioned but not of unconditioned stimuli. Psychological Review, 1980, 87, 532-552.

REBERG, D. Compound tests for excitation in early acquisition and after prolonged extinction of conditioned suppression. Learning and Motivation, 1972, 3, 246-258.

Resconla, R. A., \& Wagner, A. R. A theory of Pavlovian conditioning: Variations in the effectiveness of reinforcement and nonreinforcement. In A. H. Black \& W. F. Prokasy (Eds.), Classical conditioning II: Current research and theory. New York: Appleton-Century-Crofts, 1972.

Smirh, S. M. Remembering in and out of context. Journal of
Experimental Psychology: Human Learning and Memory, $1979,5,460-471$.

Spear, N. E. Retrieval of memory in animals. Psychological Review, 1973, 80, 163-194.

Spear, N. E., Smith, G. J., Bryan, R. G., Gordon, W. C., Timmons, R., \& Chiszar, D. A. Contextual influences on the interaction between conflicting memories in rats. Animal Learning \& Behavior, 1980, 8, 273-281.

WAGNER, A. R. Expectancies and the priming of STM. In S. H. Hulse, H. Fowler, \& W. K. Honig (Eds.), Cognitive processes in animal behavior. Hillsdale, N.J: Erlbaum, 1978.

Zentall, T. R. Effects of context change on forgetting in rats. Journal of Experimental Psychology, 1970, 86, 440-448.

(Manuscript received June 24, 1981;

revision accepted for publication January 15,1982 .) 